\title{
PV-Pix: Slum Community Co-design of Self-Powered Deformable Smart Messaging Materials
}

\author{
Dani Kalarikalayil Raju \\ Studio Hasi \\ Mumbai, India \\ daniel@studiohasi.com \\ Gethin Thomas \\ Swansea University \\ Swansea, UK \\ gethin.thomas@swansea.ac.uk \\ Adam Pockett \\ Swansea University \\ Swansea, UK \\ adam.pockett@swansea.ac.uk \\ Matt Carnie \\ Swansea University \\ Swansea, UK \\ m.j.carnie@swansea.ac.uk
}

\author{
Krishna Seunarine \\ Swansea University \\ Swansea, UK
}

krishna.seunarine@swansea.ac.uk

Yogesh Kumar Meena

Swansea University

Swansea, UK

y.k.meena@swansea.ac.uk

Jennifer Pearson

Swansea University

Swansea, UK

j.pearson@swansea.ac.uk

Deepak Ranjan Sahoo

Swansea University

Swansea, UK

d.r.sahoo@swansea.ac.uk
Thomas Reitmaier

Swansea University

Swansea, UK

thomas.reitmaier@swansea.ac.uk

Chi Zhang

Swansea University

Swansea, UK

chi.zhang@swansea.ac.uk

Simon Robinson

Swansea University

Swansea, UK

s.n.w.robinson@swansea.ac.uk

Matt Jones

Swansea University

Swansea, UK

matt.jones@swansea.ac.uk
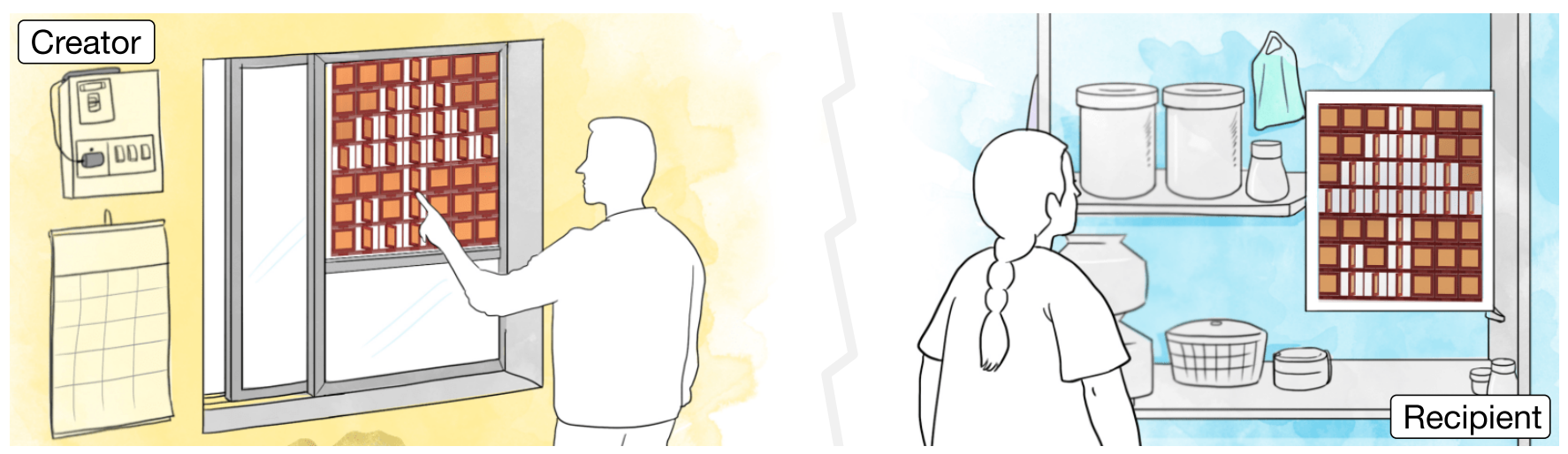

Figure 1: PV-Pix smart messaging materials in use. The sender sketches a message using a PV-Pix device mounted in a window. The remote recipient in another house interprets it as reminder to carry an umbrella.

\begin{abstract}
Working with emergent users in two of Mumbai's slums, we explored the value and uses of photovoltaic (PV) self-powering digital materials. Through a series of co-design workshops, a diary study and responses by artists and craftspeople, we developed the PV-Pix concept for inter-home connections. Each PV-Pix element consists
\end{abstract}

This work is licensed under a Creative Commons Attribution International 4.0 License.

CHI '21, May 8-13, 2021, Yokohama, Japan

(C) 2021 Copyright held by the owner/author(s)

ACM ISBN 978-1-4503-8096-6/21/05.

https://doi.org/10.1145/3411764.3445661 of a deformable energy harvesting material that, when actuated by a person in one home, changes its physical state both there and in a connected home. To explore the concept we considered two forms of PV-Pix: one uses rigid materials and the other flexible ones. We deployed two low-fidelity prototypes, each constructed of a grid of one PV-Pix type, in four slum homes over a four week period to further understand the usability and uses of the materials, eliciting interesting inter-family communication practices. Encouraged by these results we report on a first-step towards working prototypes and demonstrate the technical viability of the approach. 


\section{CCS CONCEPTS}

- Human-centered computing $\rightarrow$ Interaction techniques; Participatory design; Collaborative and social computing devices; Field studies.

\section{KEYWORDS}

Self-powered devices, Internet of Things, sustainability, connected home, interaction design

\section{ACM Reference Format:}

Dani Kalarikalayil Raju, Krishna Seunarine, Thomas Reitmaier, Gethin Thomas, Yogesh Kumar Meena, Chi Zhang, Adam Pockett, Jennifer Pearson, Simon Robinson, Matt Carnie, Deepak Ranjan Sahoo, and Matt Jones. 2021. PV-Pix: Slum Community Co-design of Self-Powered Deformable Smart Messaging Materials. In CHI Conference on Human Factors in Computing Systems (CHI '21), May 8-13, 2021, Yokohama, fapan. ACM, New York, NY, USA, 14 pages. https://doi.org/10.1145/3411764.3445661

\section{INTRODUCTION}

Digital futures are commonly imagined and constructed with communities in co-design processes [18, 19, 29]. Predominantly these activities involve what might be called mainstream or conventional users who have a relatively high degree of access and literacy in terms of digital technologies. In contrast, emergent users [7], based in the global south, have been relatively marginalised from "future making", overlooking their rich perspectives, experiences and resource constraints that can diversify and challenge the Californiancentric design standpoint [25]. Over recent years, there has been a growing interest in working with and for such communities not simply to address the challenges and needs such groups pose but also to innovate freshly, bringing new perspectives on future digital devices and services applicable globally.

Over many years of working with these sorts of communities, a common feature we have observed has been underdeveloped energy infrastructures exhibited in either a lack of grid connectivity, interruptions to supply, or simply a paucity of power points to recharge the ubiquitous mobile phone. Although not explicitly designed for such contexts, self-powered devices and services such as those proposed in $[1,8]$ seem to provide partial solutions to these issues. However, instead of seeking to deploy such proposals in an emergent user context to solve a problem, we set out to actively and deeply engage with people living in large slum communities ${ }^{1}$ in Mumbai, India, openly to explore interaction forms that go beyond those that have been imagined by conventional users [21].

Our starting point was the energy harvesting material most commonly proposed in self-powered digital interfaces - photovoltaics (PV). While many previous studies have shown the potential of using PV to drive a low-energy digital display-such as those using E Ink-or as a standalone self-powered sensor for user input, our co-design led to a disruptive proposition: why not construct a display where each of its elements-the "pixels"-are constructed of and provided energy to function by PV material. Figures 6 and 7 illustrate two types of PV-Pix envisaged (and later created) by our

\footnotetext{
${ }^{1}$ We use the term slum descriptively to draw attention to the lack of infrastructure and harsh physical realities that residents face. We distance ourselves from any normative connotations the term carries, particularly surrounding the legitimacy of land use.
}

work; one made using rigid PV material and the other flexible, rollable PV. This paper, then, contributes a novel class of self-powered deformable interfaces, showing how our co-designers led us to the innovations through sharing their communication and information needs alongside the materials and physical environments that matter to them.

As well as this material innovation, we also present one form of its use - to construct grid-like pixellated displays to afford interfamily communications. We studied the ways such displays might be used by deploying low-fidelity prototypes in two pairs of connected households over an extended period, work that highlighted the value of the material as a basis for creative, reflective message construction and interpretation. By creating fully functional PVPix, we also provide evidence that such a concept can be realised in practice and provide technical designs and starting points for others to build on.

\section{BACKGROUND}

Technologically our research is closely aligned with research into self-powered devices. Once freed from the constraint of plugging in or swapping batteries, such 'self-sustaining' technologies, in Abowd's view [1], are key to unlocking a far-reaching vision of computing where we stop thinking about digital devices and physical objects as separate entities. Such computational materials have the potential to inextricably link physical and digital entities.

The work of Grosse-Puppendahl et al. [8] shows how this vision is already being realised: that it is possible to give physical presence to snippets of digital information-such as the outside temperature or an unread message count-in the form of a self-contained, self-powered situated display about the size of a sticky note. They achieve this by sandwiching energy-harvesting PV material on the back side with low-energy displays on the front side and wireless communication in between. Their work further shows how operating under the constraint of self-power meant using lower-resolution monochrome displays as they are more energy efficient, and how different office lighting conditions (near a window vs. on a desk) affect how many times the situated displays can update throughout a day. Low-power display technologies such as E Ink and electrochromics are particularly suitable to self-powered operation as they only require power to change (rather than maintain) their display.

More recent work has shown the benefits of leveraging PVmaterials for more than energy capture. Zhang et al.'s OptoSense demonstrates how everyday objects can be extended with PVmaterials arranged along different dimensions that can be leveraged to sense changes in ambient light intensity as well as power microcontrollers to infer user interactions from changes in light intensity, such as moving a hand over an object [40]. Meena et al.'s PV-Tiles develop similar sensing abilities, but also demonstrate that organic PV materials (OPV), which can be semi-transparent or coloured, can be laid down in geometric patterns to offer aesthetic qualities much like that of a patterned tile [21]. Whereas Grosse-Puppendahl et al. [8] separated out PV from display, the semi-transparent OPV material of PV-Tiles can be layered on top of a display, which is further layered on top of microcontrollers and wireless communication modules. OPV has the further advantage of being flexible as well as semi-transparent - unique properties that Landerer et al. exploit 
in developing their self-powered sunglasses with semi-transparent and flexible OPV lenses that can also display information [15]. However, the trade-off is that semi-transparent OPV absorbs less light and is therefore less efficient than its opaque counterparts.

Dye-sensitised solar cells (DSSCs) are a different type of PV that can work well in low-light conditions and can be dyed in vibrant semi-transparent colours. Sony demonstrated these two properties in a beautifully designed solar window depicting flowers ${ }^{2}$, although their early demonstrator did not integrate the interactive capabilities of more recent research. Manufactured into the shape of textiles, Wen et al.'s research shows how DSSCs can be woven into clothing to power novel wearable experiences [39]. Wen et al. further show how more electricity can be harvested if DSSCs are combined with triboelectric nanogenerators for random body motion energy.

Although the empirical focus of our research is on PV materials and their self-powered, interactive applications, devices that leverage the aforementioned nanogenerators $[5,32]$ and techniques such as passive communication through FM backscatter [2, 36] further demonstrate a nascent research interest that is coalescing around integrating energy harvesting, sensing, computing, and communication. These are the defining properties of Abowd's computational materials [1]. So, if we follow this eponymous and ambitious vision of computing, such computational materials-produced at low-cost and scale-will create a future where "trillions of independent objects will cover a majority of the surfaces that surround us" [1].

Donald Schön famously characterised design as a reflective conversation with materials [30]. With the computational and selfpowered materials of our research thus introduced, let us consider aspects and dimensions that equally influenced our design approach and the PV-Pix concept.

\subsection{Innovation perspectives beyond the mainstream}

Reflecting on Abowd's computational materials we can't help but wonder if it is not the innovation and future-making practices that create such technological possibilities that Suchman took issue with as she penned her notes from the hyperdeveloped world [35]. To his credit, Abowd also recognises that advances in computational materials will require creative designs that reflect and emphasise societal values to "concretely influence" the capabilities of computational materials beyond their current technical emphasis. However, for this nascent field of research this has not happened yet, and is a crucial gap we address in our research.

To be sure, prototypes to control audio playback using nothing more than a drawstring of a hooded sweatshirt [32], or sunglasses with PV lenses and embedded displays [15], certainly evoke images of 'silicon valley cool'. And they certainly function as technological proofs of concept, but following Suchman we also see them as 'proof' of specific information flows - the conditions of possibility for imaging, designing and developing new technologies. It is the asymmetric nature of these flows, wherein "those in the hyperdeveloped countries maintain a disproportionate hold over the distribution of [...] information technologies" that Suchman takes issue with [35]. A powerful tactic that she suggests is to ask the

\footnotetext{
${ }^{2}$ See: https://newatlas.com/sonys-solar-windows-eco-products-2010-tokyo/17257/ (accessed July 30, 2020)
}

question: "What are the opportunities to interrupt these asymmetric flows and redirect them?" [35]. Within the arena of mobile computing, the work of Jones et al. [12] and Robinson et al. [27] suggests that innovative, radically different, and widely applicable technologies are possible if we situate design outside of the hyperdeveloped world and engage emergent users in the design of future technologies. By attending to Suchman's provocation, and situating design activities in slum communities, we also respond to Abowd's question surrounding 'core societal values', by embedding human values of diversity and inclusivity in our research.

\subsection{Dharavi}

Home to around a million residents, Dharavi is a $2 \mathrm{~km}^{2}$ slum within the city of Mumbai, India. Migrants from all over India initially settled in the area that is now known as Dharavi because market demand for its marshland was very low [4]. With little government investment or planning, and few residents having formal titles on their homes, even as early settlers improved the land and built and developed infrastructure, the cost of living remained low. Over many years, settlers developed thriving fishing, tannery and textile industries, as well as supply chains and satellite businesses. With the land surrounding Dharavi now formally developed, the slum has become extremely densely populated, with around 20 times the population density of London, or 500 times the density of Miami, by some estimates [3]. Because of this, and the growth of industry in the area, typically separated industrial, retail and residential buildings have been merged into mixed-use buildings and spaces that epitomise Dharavi today. An underutilised workshop is often transformed into a hostel or appended with a storefront, and homes also often double as shops. While some see the slum as a blemish or empathise with the poor living conditions of its residents, others see a thriving 'city-system' that while formally unplanned is inarguably designed by its entrepreneurial migrant-settlers [3].

With urbanisation on the rise and a dearth of affordable (formal) housing, especially in the cities of the global south, residents in Dharavi are living through and tackling the challenges that, as urban planners predict, people will increasingly face in the future [6]. The same can be said of Dharavi's unreliable electricity supply. Energy grids all over the world are struggling to cope with integrating renewable energy supplies [34]. From the perspective of those maintaining electrical grids, low-carbon renewable electrons are often generated at the wrong time and in the wrong places, compared to the steady and predictable ones generated through coals fires or nuclear chain reactions [34,37]. Intermittent power supply and overloaded demand, such as that residents in Dharavi routinely cope with, are part of global energy futures (including in hyperdeveloped countries) [37]. Imagining and prototyping systems that reframe energy relationships in communities at the periphery is therefore a powerful and widely applicable design method, as argued and demonstrated by Watts et al.'s 'reconstrained design' [38].

For a nascent field of research that has not quite figured out what computational materials are and, just as importantly, why they matter, we can think of no better place than Dharavi to situate our research. This is a key motivation for why we continue longstanding engagements with community members in Dharavi. 


\begin{tabular}{|c|c|c|c|c|c|}
\hline Timeline & 6th March 2020 & 8th \& 10th March & 11th March & 8th - 13th June & 20th July - 17th August \\
\hline Activity & $\begin{array}{l}\text { Community centre } \\
\text { workshop }\end{array}$ & $\begin{array}{l}\text { Workshops with artists } \\
\text { and craftspeople }\end{array}$ & In-home workshop & $\begin{array}{l}\text { Diary study in Dharavi } \\
\text { homes }\end{array}$ & $\begin{array}{l}\text { Deployment of low-fidelity } \\
\text { prototypes }\end{array}$ \\
\hline Description & \multicolumn{2}{|c|}{$\begin{array}{l}\text { Probes and discussions around self-powered } \\
\text { technologies in public and home settings }\end{array}$} & $\begin{array}{l}\text { Discussions specific to } \\
\text { home settings }\end{array}$ & $\begin{array}{l}\text { Remote video study of } \\
\text { four homes }\end{array}$ & $\begin{array}{l}\text { Intra-family trial with a focus on } \\
\text { communication and messaging }\end{array}$ \\
\hline Design insight & \multicolumn{2}{|c|}{$\begin{array}{l}\text { Focus on domestic uses rather than public } \\
\text { space use-cases }\end{array}$} & $\begin{array}{l}\text { Requires flexible, intergen- } \\
\text { erational application areas }\end{array}$ & $\begin{array}{l}\text { Windows \& curtains iden- } \\
\text { tified as important areas }\end{array}$ & $\begin{array}{l}7 \times 7 \text { grid pictograms support } \\
\text { human expression }\end{array}$ \\
\hline Covid-19 status & \multicolumn{3}{|c|}{ Before Covid-19 lockdown in India } & Unlock 1.0 & Unlock $2.0-3.0$ \\
\hline
\end{tabular}

Figure 2: Activity flow of the work presented in this paper. The overlap of the Covid-19 pandemic is also shown - "Unlock" 1.0, 2.0 and 3.0 are periods of reduced restrictions.

\subsection{Emergent users}

Residents in places like Dharavi are sometimes referred to as 'emergent users'; people who are embracing technologies such as the mobile phone, but who "may have less education [...] who may be poor [...] are often located away from commercial and political centres" [7]. Following this characterisation, research involving emergent users often seeks to address the cognitive barriers that users face in effectively utilising digital technologies [13], the implications this has for user interface design [23] and the social workarounds people deploy to access digital information, such as intermediation [28]. While we are aware and mindful of these issues, they were not an explicit empirical feature of our work.

\subsection{Sustaining human connections}

Our research with families in Dharavi, culminating in the PV-Pix concept that we present, shows how self-sustaining PV materials can also mediate and sustain human connections. CHI proceedings of the past 30 years have included an impressive and creative array of situated, sometimes tangible, interfaces to enable communication between family and friends in different places: too many to attempt to summarise here. We note, though, that we could not find any designs or systems that operate under the constraint of self-power. So we instead draw upon Harper's reflections on the philosophical and empirical fallacies implicitly embedded into the design of many computer mediated communications systems: namely, that 'more' is better [10]; that synchronous is better than asynchronous; that visual is better than auditory; and, that multisensual is better than unisensual. Similarly, when considering the technical implementation of these communication channels, Harper warns us of the metaphysical trap of thinking of human communication in a mechanical sense: that it is an exchange of information or messages passed back and forth [10]. Adopting such a view comes at the expense of the much harder to cultivate sensibility that sees the performative values as well as the social and moral implication of communication. However, adopting a performative view of communication and operating under the constraint of self-power draws attention to the ways in which simpler interactions can be leveraged for creative expression and ultimately to create and maintain social bonds between families. One suggested strategy is to think of the messages communicated between close relations as gifts [10].

\subsection{Methods}

Working, as we did in the work reported here, across cultures, geographies and disciplines, with all its attendant challenges and injustices, highlights that the world we live in is a mess - to paraphrase the social scientist John Law [16]. The research activities reported here were also undertaken in the time-period immediately following the start of the global Covid-19 pandemic, and carried on during the months of lockdown in Mumbai, in an unfamiliar, remote form (see Fig. 2). Our research therefore resonates with Law's suggestion to stop thinking of a method as a form of black box, to be taken off a shelf, and where new inputs yield new outputs. Methods, in Law's view, should be carefully adapted and 'assembled' and subsequently performed in response to the specific social, technical, environmental, or public health 'mess' at hand [16].

In reality, then, the PV-Pix concept is not the outcome of a particular method, nor is it attributable to a particular participant or researcher. It is the product of entangled engagement with people, materials, place, and diverse perspectives. That said, initial workshops (Sections 3.1 and 3.2) were planned based on previously published emergent user community methods, including the value of enriching these with artistic perspectives as described in [24-26]. The results from thematic analyses of these two initiators and the home workshop (Section 3.3), led to a diary study that in turn highlighted the opportunities embodied in subsequent low-fidelity prototypes.

\section{RE-IMAGINING BUILDING MATERIALS IN SLUM SETTINGS}

We began our engagement with the Dharavi community by introducing the concept of self-powered digital materials to a community leader who, over the course of the research, became a valued community liaison. With his help, we surveyed the built environments in Dharavi and found that tiles are not only a pervasive and aesthetically decorated building material, but also one that is manufactured locally by potters who have been working in Dharavi for over a century [14]. This suggested that the PV-Tiles concept, as developed by Meena et al. [21], would be a fruitful starting place to explore and co-design self-powered computational materials that could be embedded into Dharavi's built environment and potentially even manufactured nearby. The PV-Tiles concept closely couples the energy harvesting material with an interactive display and sensors into a tile module so that self-powering interfaces can be fully integrated with surfaces as diverse as kitchen walls, floors and tabletops.

While the liaison recruited participants, spoke with potters and found space in a community centre for us to host a design workshop, the team of researchers developed probes-strategically incomplete prototypes-to surface specific material constraints or material 

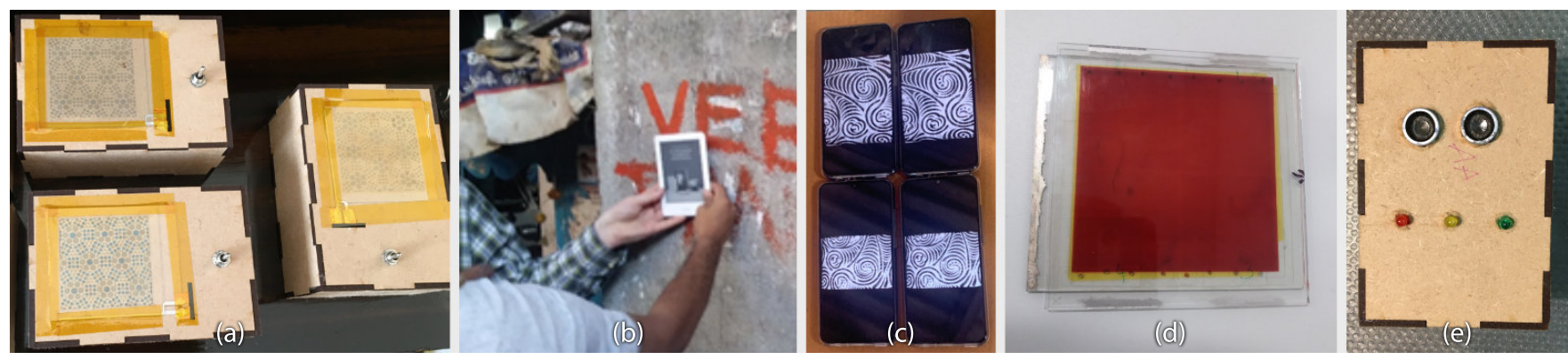

Figure 3: The technology probes we used in the community workshop in Dharavi. From left to right: (a) electrochromic displays, (b) E Ink displays, (c) connected phone array, (d) dye-sensitised solar cell (DSSC), (e) proximity sensor with LEDs.

properties of self-powered interfaces and interactions as suggested in [21]. Our co-design workshops with residents of Dharavi, therefore, became a dialogue of people, place, and self-powered digital materials [30]. Over the course of five days we conducted two halfday workshops in Dharavi, one with residents in the community centre, and another in a participant's home. We also extended design activities between those two workshops, by visiting a potter's manufacturing workshop in Dharavi and by engaging with artists local to the Mumbai area. So by the time of the home based workshop, we had gathered further design materials and refined probes to deepen our engagement and dialogue with earlier participants.

\subsection{Envisioning workshop with the community}

We recruited 12 emergent user residents of Dharavi (6F, 6M) aged (18-64) broadly representative of the Dharavi population who resided in tightly-packed one- or two-room dwellings and lived with daily resource constraints including limited access to sanitation, electricity and internet. The workshop was organised and attended by six of the team consisting of designers, computer scientists, electrical engineers and translators; took place in a community hall in Dharavi; and, like all activities reported here, began with an informed consent process for which we obtained IRB ethical approval. Each participant was paid ₹500 ( $\approx$ \$) for taking part.

We brought five probes to surface and demonstrate the material properties and constraints of technologies that could potentially be self-powered. The probes were (as shown in Fig. 3): (a) a transparent electrochromic (EC) display which can shift between two geometric patterns of roughly equal size; (b) E Ink devices to display a collection of tile patterns and images captured in Dharavi; (c) a connected array of smartphones with greyscale filters, where changing or interacting with an image on one phone would change the whole array; (d) a DSSC cell to demonstrate how solar cells can be semitransparent and colourful; and, (e) proximity sensors connected to LEDs - moving a hand close to the device turns on the lights.

We explained that E Ink and EC displays are good candidates for self-powered displays as they only require power when changing the displayed image, but could not be updated quickly as it takes a second or two for the displays to change state (EC) or show the next pattern (E Ink). By demonstrating these unfamiliar display technologies, we asked participants to reflect on the qualities of the EC and E Ink displays, especially in comparison to the television or mobile displays participants were familiar with. In the case of EC displays and DSSCs-two semi-transparent materials-we further demonstrated how these two technologies could be merged together to create self-powered displays, with the DSSC overlaid on top of the EC. Finally, we also showed how such self-powered tiles could be extended through sensors to drive interactivity, as demonstrated through the proximity sensor, or connected to nearby 'tiles', demonstrated through the phone array.

Discussions surrounding the individual probes, at the start of the workshop, showed that participants understood the concepts and constraints behind each of them and the overall aims of the PV-Tile concept [21]. After this introductory session we asked the participants to consider the use of PV-Tile-like modules in home and public settings. This brainstorming session led to ideas and scenarios that were scaffolded by participants' familiar experiences with their mobile phone and through conventional uses of PV panels. For instance, in thinking about ways in which EC displays could sense and react to gestures, participants drew upon the familiar gesturebased user interface of the mobile photo gallery. They suggested how users could swipe through and (pinch to) zoom into photos displayed on a tile. Or, in discussing how DSSCs could power a tile, participants expressed a desire for DSSCs to power or charge other devices. In discussing these examples we do not intend to critique participants; to the contrary, we are thankful for their creativity and dedication. If anything, these cases show that the probes we used, which presented the display as a separate component to the energy harvesting surface and sensors, did not afford divergences from "conventional" self-powered interfaces such as those previously reported by [21]. This failure in innovation-reported here in the spirit of "documentation of process", typical in design-oriented research [41]-was a first spur to reimagining the role of energy harvesting elements that was elaborated through the further studies presented later in this paper.

Turning now to potential applications, those relevant to inside homes for members of the family dominated discussions in comparison to public and retail settings that were also explored. For analysis, researchers reviewed videos and notes independently, initially through the lens of the possibilities afforded by the different probes. This first stage was then complemented by a group thematic analysis identifying the unprompted applications and material integration. The results strongly indicated home use over public use with few suggestions around applications outside the home. The analyses from this session revealed a common desire to use the technologies to connect to specific people, places, and deities. For 
instance, one participant mentioned she would like tiles to represent the landscape of her village, which prompted discussions around connecting with family 'back home' in the rural areas they had migrated from. The eldest participant suggested messaging a tile with her location to update her grandchildren on her whereabouts. Using the tiles to keep neighbours in contact was also highlighted as valuable.

Thinking about how tiles could be integrated into existing visual elements in their homes, there was agreement amongst participants that a tile could show an image of a God most of the time, but then switch to a different image to signify a particular event. These triggers ranged from a child returning from school (represented by an image of a bus) or the occurrence of a festival that would change the default image to one more closely associated with that event.

After the community centre discussions, the group split into two with each sub-group accompanied by three of the research team. These groups then carried out a walking technology tour [33], identifying places in the narrow alleyways and wider main streets for possible uses of self-powered surfaces. Common suggestions included kiosk shutters to show opening hours; integration with tiled walls to display community information; and, door-steps for tiles that could display changing Rangoli-style patterns ${ }^{3}$ or enable simple games. However, there was also a shared concern about the security and robustness of public deployments, with both groups suggesting the need to place devices out of reach to avoid theft or damage.

While most of the workshop was either in an uncommonly open and airy room or out in the streets of Dharavi, upon concluding the workshop one participant invited the researchers into her nearby single-room home so we could see for ourselves the densely populated and multi-purpose context that any home-based self-powered design propositions would need to be situated within.

\subsection{Session with artists and craftspeople}

Following the community-centre workshop, we subsequently engaged with three artists $(2 \mathrm{M} \& 1 \mathrm{~F}$, each paid ₹2,000 $(\approx \$ 27))$ from across Mumbai to gather feedback on the technological possibilities of self-powered interactive materials in general and on the themes and application areas that emerged from the community centre workshop specifically. Furthermore, as an expressive exercise we also asked the artists to sketch interactive tile concepts for us to showcase to community centre workshop participants. One of the artists who practices ceramic glazing worked with another who was an animation artist, and prompted us to think about how selfpowering interactive materials could be leveraged for movement and effect in an otherwise static built environment. The concept they developed was a single line of tiles each consisting of an energy-harvesting DSSC outer layer, sitting above an EC display that could switch between two states: empty sea or a ship on the wave. By switching state across the line of tiles in a room, the boat could appear to move along the waves as the day progresses, with users hanging things on the wall to represent activities that need to be done at particular times of day.

Given his experience in architecture, the third, senior and nationally recognised artist prompted us to think about materials

\footnotetext{
${ }^{3}$ Rangoli is an art form in which intricate patterns are made on the ground using colourful powders.
}

beyond tiles: doors, windows, and other thresholds; key insights, as it turned out, for the PV-Pix concept that we present later in this paper. Drawing on his interest in aesthetics of specific Indian cultures, he showed us how good humour and religious imagery could be integrated to facilitate intergenerational communication within a family. For his specific example, he sketched a tile showing a smiling Hanuman (a well-known Hindu God). As a way of telling his children to clean the room, he could gesture at the tile, causing Hanuman to close his nostrils with his fingers because of the smell in the room. Once the task was complete, the children could gesture at the tile and make Hanuman smile again.

To supplement this artistic perspective, we visited a renowned potters area, situated less than 200 metres away from the homes of the emergent user community members who attended the workshops, and engaged five craftspersons living in Dharavi. Craftspersons then made tiles out of terracotta clay depicting ideas from the workshop and engaged in discussions with the researchers, reflecting on both the new market possibilities of adding modern digital materials to ancient clay practices, as well as noting the practical difficulties of doing so.

\subsection{Co-design workshop in the home setting}

Since domestic settings and inter- and intra-family communication scenarios were key themes in the community centre workshop, we asked the community liaison if it was appropriate for us to have a follow-up workshop inside a home. One participant volunteered her home, and together with the liaison she agreed to host a half-day workshop with three researchers (designer \& translator, computer scientist, electrical engineer) and five female participants from the earlier workshop, including herself. We paid each participant ₹500 $(\approx \$ 7)$ for their participation in the study.

Inside the home the idea of a self-powered device resonated with the participants, who shared their difficulty in getting access to a charging point, as homes usually have only one or two wall sockets. Participants were shown the artists' sketches, the clay tiles produced by potters, and further sketches we prepared after the previous workshop based on ideas it generated. This led to interesting discussions that, situated inside the home, ultimately, critically interrogated the tile concept as a whole. When asked to imagine living with different tile concepts, participants expressed concerns surrounding application areas that are too narrowly defined or are tailored to only one person. They also wondered how durable a tile could actually be - a valid concern. Taken together, these discussions suggest that the PV-Tile concept as seen in previous work is too strongly fixed within the environment, and too tightly integrated and focused on specific, rather than flexible application areas. Alongside this important insight, by situating this workshop inside a single-room home, we were exposed to how in-home constrained spaces are frequently rearranged, in this case both to accommodate our workshop and to accommodate the needs of other residents.

\section{DIARY STUDY}

Over the various community workshops and activities participants demonstrated increasing understanding of how technologies that could reasonably be self-powered worked, and saw greater value in 


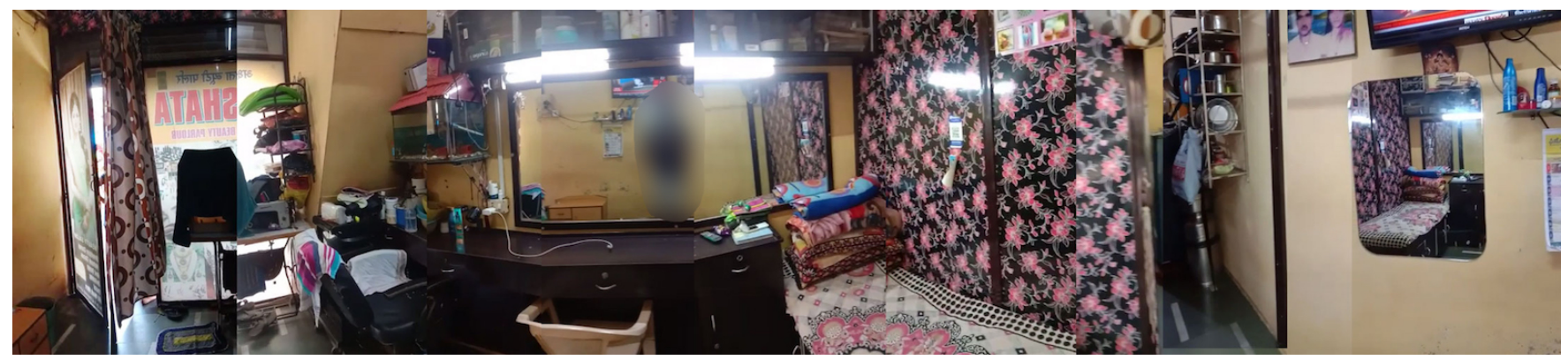

Figure 4: An example of the type of household videos submitted during the diary study (image created for this figure by merging frames from a submitted video into a single panorama image). This single room home functions as a beauty parlour by day and a family space by night.

home-based installations. However, the question of what forms and functions these self-powered technologies could take on in order to fit within the existing material and uses of a Dharavi home was never quite addressed. This suggested that we-the researchersneeded to learn more about the everyday (spatial) practices of residents living in single-room, multiple-use homes. In order to explore this aspect further we conducted a follow-up study into the uses, behaviours and adaptions seen in typical dwellings. We used a diary study methodology, recruiting households in slum areas of Mumbai to provide insight into their living spaces.

\subsection{Participants and method}

Four households (three four-person; one five-person) took part in the study. All participants were originally migrants to Mumbai from the same region, but have lived in slum areas of the city for the past 15-20 years. Each dwelling had between one and three rooms, separated either by a temporary partition or subdividing walls. All adults in the households (apart from one who is an office clerk and uses a laptop) were classed as emergent users - that is, they have low textual literacy and have difficulty in typing on a phone, preferring to use voice messages or phone calls. We followed an IRB-approved informed consent procedure with the family, mediated via the head of each household, obtaining permission to anonymously store submitted diary materials (consisting of images or videos of house interiors) and audio recordings of follow-up interviews.

The diary task was designed to capture regular snapshots of the layout, usage and any changes to the fabric of each household. In order to do this, the head of the household (or a delegated adult family member) was instructed to stand in one place, begin recording a video on their mobile phone and slowly pan to show the whole space, then move to the next room (if applicable), and repeat (see Fig. 4). Participants were asked to complete this task three times per day (morning, afternoon and evening) each day for six days. We asked participants to avoid capturing images of people, and to review videos before submitting to ensure they were comfortable sharing the content with us.

At the end of each study day a researcher interviewed the head of the family or the engaged member of that day to discuss and capture any clarifications on the use of articles and space shown in the shared media. After the third day of the study the wider research team reviewed the materials to discuss use-cases and raise any queries to feed back to participating families. At the end of the sixday diary study period we conducted a final interview session with each family to review the materials and discuss potential insights. We paid each household ₹4,000 ( $\approx \$ 55)$ for their participation.

To analyse the submitted diary materials, three members of the research team divided the incoming videos between themselves and recorded observations and insights in the following focus areas:

- Changes in usage of the space at different times of day

- Objects that are triggering changes in the sense of space (e.g., movement, addition, division, removal, etc.)

- Correlations between object usage patterns inside the homes (e.g., curtains closed to avoid glare)

- Lighting use and availability; control of light (e.g., sunlight vs. artificial light)

\subsection{Contextual insights}

In total, 65 videos were shared from the four homes. ${ }^{4}$ Following the procedure outlined above, we analysed these materials, generating a range of contextual insights that are detailed below using illustrative examples.

Space adaptations: All of the households that participated in the study had dwellings that were small in area. However, the adaptation of spaces within these constructions is a result of a lived experience of domesticity unique to its cultural context. All of the households subdivided the spaces into specific use-case-focused areas as might be expected. But there were also interesting examples of more substantial adaptations. For example, one of the homes was used both as a living space and a public beauty parlour. One partition of the single-room dwelling was used as the shop during the day and the bedroom at night. Household objects such as the television and sofa change their context in the evening as the shutter of the shop goes down and the space transitions into a private home accommodating four people. The sofa becomes a single bed, and all of the floor space is converted by laying out a foldable mattress and bedsheets. Customisations such

\footnotetext{
${ }^{4}$ In one household unavailability of a shared phone caused the participant to miss four video submissions. In another the house's dual-use as a beauty parlour meant that the participant was busy with clients and unable to create a video on three occasions.
} 
as these highlight the real need for flexible and adaptable technologies that can accommodate and be successfully used in highly multipurpose spaces.

Materials and aesthetics: As described above, participants were used to moving objects and partitioning rooms in temporary or more permanent arrangements. This was achieved in most cases by, for example, hanging a curtain on a rope suspended between nails; fixing a rod when a semi-permanent divider was needed; or, constructing a metal frame with rigid sheets of patterned material for more permanent needs. In terms of materials used for this, textiles were the most commonly seen, in addition to their usage as curtains and drapes over windows and doors.

At first glance, many of the spaces shown in participants' videos might seem cluttered, with various objects competing over the limited space available (Fig. 4). However, most of the surfaces, objects and placements were carefully considered by participants. Participants recounted in great detail the rationale and links between their wall decorations, deity images, pictures, curtains and other object used to decorate and augment the spaces. This was a clear insight that aesthetic qualities are as important as functional qualities for the proposed material.

Lighting: Light availability is clearly a key consideration when designing devices that are intended to be self-powered using photovoltaics. For our participants, availability of sunlight inside their homes varied considerably through the day. Transition spaces between the outdoor and indoor (e.g., windows and doors) were able to let ample amounts of natural light into all four of the homes.

These findings suggested two potential materials and sites of interest within the homes. Firstly, the transition spaces (doorways and windows) usually included blinds of various designs including strings of rigid glass beads or textile drapes; and, drapes were pervasively used to partition living spaces. In addition, the artists we worked with earlier identified aesthetic and cultural values of such thresholds (see Section 3.2). The prototypes simply showed these materials with representations of where an interactive element could be incorporated (e.g., within the curtain pattern or on a window slat). The comments by participants on these confirmed them as potential sites of value and interest that we explored in low- and high-fidelity prototypes.

\section{FROM DESIGN INSIGHTS TO A RE-SEEING OF PV MATERIALS AND INTERACTION}

Taking the thematic analyses from the workshops and diary study, we explored overlaps of the predominant categories. This identified the home as a key site for exploration (see Section 3); the need for a strong consideration of the materials and their home use (Section 3.2 and Section 4); and, a desire for diverse ways to maintain connections (Section 3.1)

Home as key use context: While the workshops exposed potential uses of the material in public settings, a richer set of opportunities was apparent within the home context. Partially this was due to security and robustness considerations of alleyway- or shop-front-based installations. More significantly as we moved our focus and discussions into participants' homes, it was clear that, given the small, densely and diversely populated dwellings, home dwellers gave much thought about the value of and use of materials they chose to place in their rooms. These choices have to accommodate the inter-generational occupants of the relatively very small living spaces; and, re-configurations of the space to enable different activities.

Collaging and the home environment: One of the functions of the form and use of the slum dwellings was a highly apparent mixing and embedding of materials reflecting different home tastes and needs as well as the desire to optimise the use of surfaces and other objects. Whereas multi-room homes in more affluent regions might see different aesthetics on display in different rooms or one aesthetic choice articulated in more diluted forms across several rooms, in the slum dwellings there was an intense mix of material forms, colours and their juxtapositions.

Maintaining relationships: Being connected was seen as critical to our participants, be that with the rural regions they migrated from or with family and friends a few alleys away. In terms of digital services to satisfy this need for connection, WhatsApp and phone calls were pervasive. Participants' access to these technologies was uneven, however, with the older generations forsaking WhatsApp and there often being one device shared between the entire household.

\section{THE PV-PIX CONCEPT}

Reflecting with our co-designers and considering the responses of our artist and craftspeople participants, then, we saw an opportunity for a PV material that: is home based; could be woven into the fabric of the home responding to aesthetic and spatial considerations; and, is inter-home relationship focused but provides a more inclusive and alternative to the hyper-connectivity seen with mobile messaging services.

We propose a novel and disruptive use of energy-harvesting materials to create displays for such inter-space connections. Conceptually, each display consists of one or more PV-Pix. Each PV-Pix pixel is a module that is made of materials that can harvest energy and use that power to deform itself once actuated by a person, altering its state to communicate between homes. Deformation for communication was seen as valuable as it enables the sort of non-screen display and more tangible interactions valued by participants as well as fitting the aesthetic and reconfiguration dynamics identified in the diary study.

We include this design space as one of our aims is to connect $\mathrm{HCI}$ with material science and engineering. We hope it will be used by multidisciplinary teams to create a range of displays that go beyond those prototyped here:

Energy harvesting technology: An individual PV-Pix pixel may be constructed of a number of PV technologies (e.g., dyesensitised, perovskite, organic or silicon solar cells). PV technology is developing at a rapid pace both in terms of its harvesting efficiency and deployment, with future innovations to include woven or printable PV, enabling the PV-PIX 
to be incorporated within the wide range of contexts seen in the home workshop and diary study.

Form factors: A PV-Pix pixel may be made into different shapes and sizes, may be patterned and coloured, and can have different degrees of flexibility (i.e., rigid to fully flexible). These form-factors enable PV-Pix elements that can be embedded into a range of other home materials. For example, strings of glass beads used as a door curtain in some of the diary study homes we observed could become PV-Pix displays with each glass bead being a PV-Pix element.

State change mechanism: A PV-Pix module is used to communicate in a visual way by changing its physical appearance via any mechanical manipulation technique, including angular movements like rotation or tilting; or, linear ones such as rolling or unrolling.

States: Once actuated the deformation leads to a state change. The change might be related to one or more possibilities, such as orientation, colour, pattern or image visible, revealing or hiding the aesthetic elements that are integral to the PVmaterial. This feature-and the previous one-was inspired by the range of re-configurations and re-arrangements of objects and materials seen in the diary study.

Device construction and configuration: PV-Pix pixels could be arranged in different configurations in 2D or 3D space with different spatial relationships. Most simply, the device could consist of one PV-Pix, while a simple configuration of multiple PV-Pix elements could be a grid pattern (as explored later in this paper). Consider, also, more complex arrangements for example, creating a spherical display consisting of a number of curved PV-Pix modules each rotating individually and independently to show different patterns, accommodating, for instance the non-tile examples surfaced in the workshop with the artists.

Gesture sensing: A PV-Pix pixel could sense a user's gesture from the shadow they create by blocking the incidental light, or the light reflected or shone onto it due to the user's gestures. Alternatively, additional sensors-dependent on the power available-could be used, such as proximity or touch. We note that a range of gestures and inputs (including speech) was observed in the community envisioning workshop.

Actuation techniques: A PV-Pix pixel will harvest energy to power its actuation system. A low-power and compact actuation mechanism (e.g., electromechanical) will be beneficial for a higher refresh rate and smaller form factor. A servo motor will achieve a lower rate of operation, but provide greater deformations.

Communications and electronics: In a PV-Pix device, all modules can act together as one unit by self-powering their communications using a low-power communication technology (e.g., Bluetooth, ZigBee, etc.). A low power microcontroller unit on each pixel with sleep/wake functionality for eventdriven operation will be beneficial. Alternatively, a PV-Pix device could be configured in a active-matrix arrangement for its pixels to share one or a small number of energy storage, microcontroller and communication units.
Pixel-based communications have been explored in previous work (e.g., [31]) and commercial platforms exist ${ }^{5}$. Autonomous, tangible interfaces that handle both display and interaction have similarly been explored [17]. However, in contrast to these, PVPix is self-powered; the energy-harvesting material is the display surface; and, the displays are tangible and deformable in nature.

\section{DEPLOYMENT}

In order to gather insights into how devices constructed of PVPix elements might actually be used in practice, we conducted a longitudinal trial of two low-fidelity PV-Pix designs in-situ within the homes of slum residents.

\subsection{Low-fidelity prototypes}

Using the design space above as a guide, we developed two lowfidelity PV-Pix prototypes (see Fig. 5) which we later deployed amongst residents of two slums. The objects used as starting pointswindows and curtains-were identified by our participants as important areas of their homes, and allowed us to consider different dimensions of the design space in our evaluation.

PV-Pix and window frames: Figure 5 (a) shows the low-fidelity version of the PV-Pix window prototype. The configuration was modelled on a window blind with a $7 \times 7$ grid of square PV-Pix slats of $70 \times 70 \mathrm{~cm}$ in total. Each slat-simulated here by perspex-represents a dye-sensitised, single-coloured, rigid material that would be deformed by a 90 degree turn on a dowel. The states represented were 'closed' where the PV-Pix material was fully visible; and, 'open' after the material had been rotated to reveal side view of the perspex. For the prototype deployment, actuation was done by hand by the participants as described in the deployment plan below.

PV-Pix and curtains: Figure 5 (b) shows the low-fidelity version of the PV-Pix curtain prototype. In this design, each PVPix-simulated here by coloured card-represents a flexible organic PV material that we envisage could change state by rolling up (to be closed) and down (to be open). For the low-fi prototype, these rollers were simulated by different coloured cards that could be manually flipped to indicate a state change - i.e., red here means rolled up (closed) and blue means rolled down (open). As with the window frame, a grid of 49 PV-Pix elements was created, pinned to a curtain in the participants' homes.

\subsection{Deployment plan}

We recruited the same four households $(\mathrm{H} 1-\mathrm{H} 4)$ who participated in the diary study described earlier in this paper for a longitudinal study over a four-week period (five days per week, 20 days total), providing participants with a week of each type of interaction (i.e., send or receive messages) on each prototype (i.e., window or curtain). Prior to the deployment taking place, the head of the families were verbally briefed about the purpose of the study, and digital written consent for the entire family was collected. Each household was paid ₹10,000 ( $\approx$ \$136) for their participation.

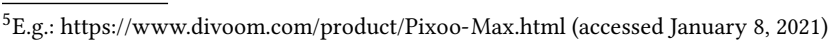




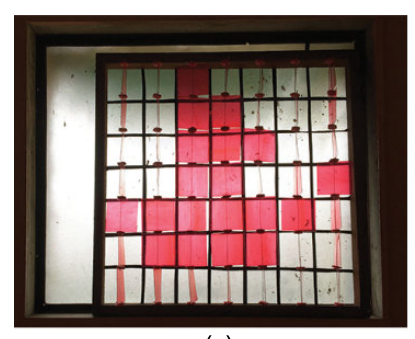

(a)

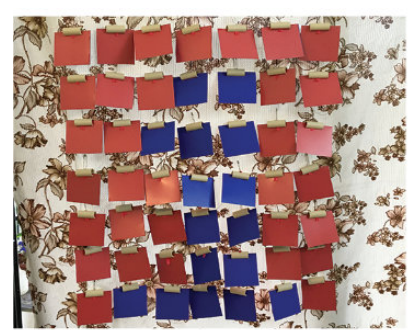

(b)

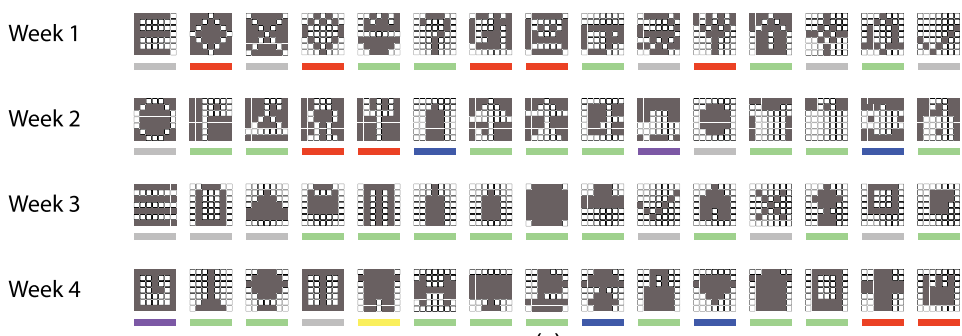

(c)

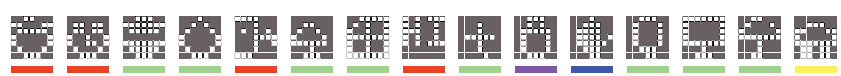

Week 2

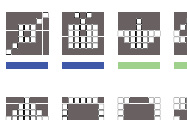

Week 3

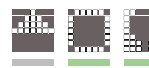

Week 4

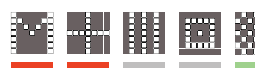

Figure 5: (a) PV-Pix and window frames low-fidelity prototype. (b) PV-Pix and curtains low-fidelity prototype. (c-d) Pictographs of all the images created/received on (c) the window frame prototype and (d) the curtain prototype over the 20 days of deployment. Note that $(c-d)$ have been colour-coded to illustrate the categorisations we have applied to each image.

The households participated in two pairs of relatives $(\mathrm{H} 1, \mathrm{H} 2$ and H3, H4), in order for us to gather information about how they might use each of the prototypes for sending and receiving messages to loved ones. Due to Covid-19 lockdown restrictions at the time of the study, we were unable to demonstrate the prototypes in person, so an instructional video was shared with all participants and further explained over phone calls at the start of the deployment. The following is a summary of the plan of the deployment:

Week 1: In the first week of the trial, $(\mathrm{H} 1, \mathrm{H} 2)$ and $(\mathrm{H} 3, \mathrm{H} 4)$ pairs used the windows and curtain prototypes respectively. (H1, $\mathrm{H} 3)$ pairs emulated sending messages and $(\mathrm{H} 2, \mathrm{H} 4)$ receiving. One of the family members from $(\mathrm{H} 1, \mathrm{H} 3)$ was instructed to create a picture message for sending with the prototype thrice a day, (i.e., morning, afternoon and evening), and sent a photo of the picture message made on the prototype with a short description to a researcher. The researcher then forwarded the photos to $(\mathrm{H} 2, \mathrm{H} 4)$ without the message description. One of the recipient family members then recreated the picture message received and tried to guess its meaning, and sent back an image of the recreated picture message captured using their mobile phone, along with a description guessing what the sender might be trying to communicate each time. The researcher then called an adult in the sender and receiver families to discuss the messages from that day.

Week 2: In the second week the households swapped their roles; that is, $(\mathrm{H} 2, \mathrm{H} 4)$ and $(\mathrm{H} 1, \mathrm{H} 3)$ pairs emulated sending and receiving messages respectively.

Week 3-4: In the third week, the households swapped prototypes and roles. In the fourth week, they swapped their roles but kept the same prototype. In this way, all households took turns at sending and receiving messages with both prototypes. In this second half of the study the participants and researchers followed the same protocol as before, (i.e., with daily updates and weekly interviews). However, at the end of fourth week there was an additional interview discussing the experience over the entire four weeks of deployment.

\subsection{Results}

Over the course of the four-week trial, participants created and interpreted a total of 120 messages (60 messages per prototype). Since each message and interpretation was 'relayed' through the researcher, we developed an understanding of each message's content and, more importantly, its significance over time. We further discussed each message during bi-weekly meetings with the wider research team. We found Hassenzahl et al.'s classification of the strategies used to create experiences of relatedness to retain at least some of the relational significance of the messages that households created for each other [11]. In Fig. 5 we show pictographic representations of the messages created on both systems and, following Hassenzahl et al.'s method, broadly classify these as: awareness (50 $\%)$, expressivity (17\%), joint action (7\%), gift giving (6\%), memories $(2 \%)$, and other $(18 \%)$.

After analysis of the photos, descriptions and interpretations sent to the researcher by both home parties, we concluded that participants identified the visual image correctly $68 \%$ of the time, whereas interpretation of the underlying meaning of the message was correct $52 \%$ of the time. We also deduced that $34 \%$ of sent messages were not intended to contain any underlying meaning. These "non-meaning" messages, however, were predominantly sent during the initial weeks of the study. Messages created towards the end of the trial tended to be more nuanced, indicating that the forms of expressions enabled by the prototypes evolved through use [10] and resonated with the principles of 'slow technology' [9]. 


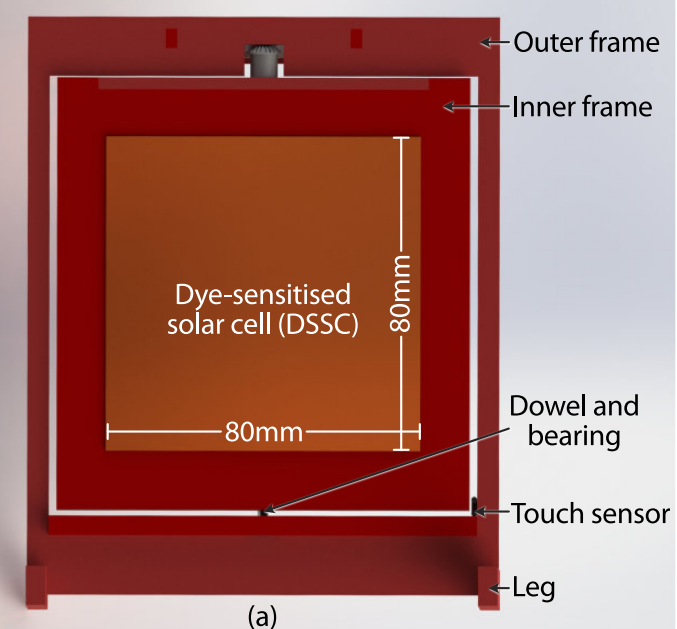

(a)
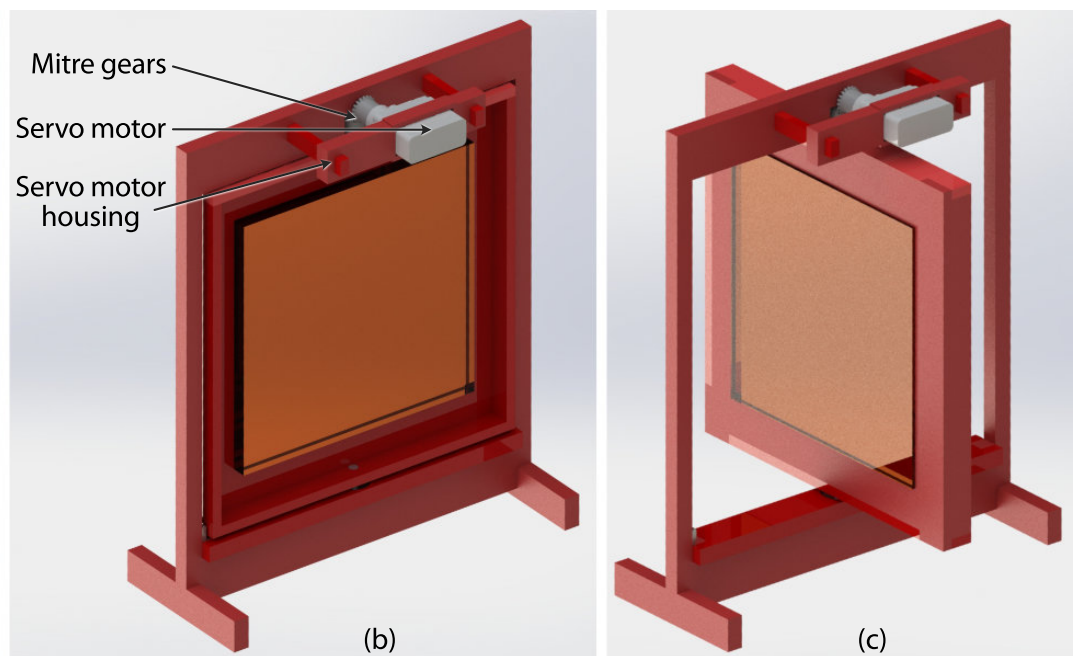

Figure 6: PV-Pix prototype 1 - a single TiltTile module that can be composed into a larger grid of identical elements. The device is constructed using a DSSC fabricated in our lab, and is used for a rigid-type deployment (e.g., in a window). From left to right, the three views show the device's (a) construction, (b) closed state and (c) open state. Note that we use rendered diagrams of the prototype for clarity here. The accompanying video figure shows working examples of the actual prototype in use.

In fact, the biggest challenge surfaced by participants was that the transfer of the rich information in a message framed in the mind-usually in the form of a sentence or phrase-was sometimes lost on the encounter with the limited possibilities of a 49-pixel square. They discussed that drawing and visualisation skills are necessary to make these messages - one participant during week one shared his strategy of making the message on paper as a line drawing over 49 hand-drawn squares before replicating it on the prototype. Despite these issues, participants managed to express and interpret detailed messages to one another during the course of the deployment, which has given us some insight into how and why such interfaces may be used in these contexts. Below we discuss a summary of some of the more notable scenarios surfaced:

Gifting a lamp: One evening, there was a 30 minute power breakdown in the area because of the monsoons. During the breakdown Kaveri ${ }^{6}$ (H1-F14) thinks about a message to send to $\mathrm{H} 2$. She remembers the conversations between her mother and Tulsi (H2-F33) about Shekhar (H2-M40) working late into the night as he juggles two different jobs. She decides to make a picture message of a lamp, and starts rotating the PV-Pix elements on the window. After getting positive feedback from her brother, Krishna (H1-M16), she takes a photo and sends it to the researcher, along with the description "Table lamp - for Shekhar to use as he works late in the night - Kaveri". Shekhar, on receiving the image (minus the description), goes to his window and begins recreating the picture message. Once completed, he sends a photo with a description "Looks like a table lamp, could be used at night" to the researcher. He later receives a reply from the researcher stating: "Congrats, you interpreted it correctly. Kaveri sent this to you to use it while working at night".

${ }^{6}$ Names used are changed for anonymity; household number, gender and age are indicated in parentheses.
Umbrella reminder: On another morning, Anand (H1-M38)-a street vendor-was having chai at his home. The sun was behind dark clouds and reminded him of the previous evening when he was stranded in front of a shop waiting for the rain to subside. He told his daughter and wife that if he had taken an umbrella with him, he could have been back home earlier last night. His wife, Yamini (H1-F36) suggested to her daughter to send a message of an umbrella to H2. Her daughter quickly turned the PV-Pix pixels on the curtains and shared the image with the researcher with a description "An umbrella for the rain - idea by Anand, made by Yamini and Kaveri". On receiving the image forwarded by the researcher, Shekhar (H2-M40) recreated the message on his window and responded: "Umbrella - it might rain, use an umbrella - Shekhar".

Life insurance payments: Another night, Yamini (H1-F36) was worried about missed payments to LIC (Life Insurance Corporation of India), a small monthly investment she has been making for her children. She remembers how her children previously created a message containing the letters L-I-C for Shekhar (H2-M40), who works as an LIC agent and recognised the letters. The next morning, she created a message containing a question mark and hoped that Shekhar would understand she was asking about the next due date. She sent a photo to the researcher. On receiving the relayed image, Shekhar replicated the question mark and sent an image of it with the caption "Possibly asking the next due date for LIC".

These scenarios show how expressive participants can be even with the simplest of abstract images, particularly if the messages are-like these examples-exchanged between close-knit friends and relatives who migrated to Mumbai from the same area 15-20 years ago and maintained regular contact and care for each other. 


\section{PV-PIX PROTOTYPES}

In order to demonstrate the feasibility and viability of the PV-Pix concepts explored in the low-fidelity prototype study, we built two exemplar hardware devices: TiltTile (illustrated in Fig. 6) and FabricOn (shown in Fig. 7). Both prototypes are shown in use in the accompanying video figure. In the rest of this section we outline the hardware and electronic design and construction of these devices, and demonstrate their ability to provide self-powered remote communication.

\subsection{TiltTile}

The TiltTile hardware demonstrates remote picture messaging using a rigid PV-Pix device employing DSSC technology and designed to be deployed on a window. To create a TiltTile module we fabricated custom DSSC cells in our laboratory following the processes described by Solaronix [20] without the need for a clean-room environment. For each cell we used two fluorine-doped tin oxide (FTO) coated glass substrates with a thickness of $2.2 \mathrm{~mm}$ as the top and bottom electrodes, and a screen-printing technique to deposit a layer of titanium oxide on the bottom electrode, sensitised using ruthenium dye. After cleaning, the top electrode was spin-coated with a platinum precursor and dried on a hotplate The two electrodes were then sandwiched together around a gasket before being filled with electrolyte and sealed to form an $80 \times 80 \mathrm{~mm}$ cell.

The DSSC cell is mounted inside an acrylic inner frame with mitre gears at the top and bearings at the bottom (Fig. 6 (a)) and electrical pressure connectors on the sides, extended beside the gears. This component fits into a larger frame (see Fig. 6 (b)) to create a single TiltTile module. A servo motor is used to rotate each solar cell around its vertical axis (Fig. 6 (c)), with an MPPT energy harvester/charger board, lithium-ion battery, microcontroller and XBee wireless communication board for control and remote connection. A discreet, lab-made touch sensor is mounted on the frame to provide interactive input. We built two $2 \times 2$ configurations of these modules to demonstrate the design's ability to provide remote communication as explored in the low-fidelity prototype study. When an individual TiltTile is touched, it rotates from the closed to open state (or vice-versa), shortly followed by its remote counterpart.

\subsection{FabricOn}

The FabricOn hardware uses an organic photovoltaic (OPV) cell, and is deformable, designed to be mounted over a curtain. In this demonstrator, then, a FabricOn module changes its state by rolling or unrolling a section of PV tape. To achieve this we used commerciallyavailable flexible bidirectional solar cells of 110x160 mm which are mounted on a 3D-printed casing, as shown in Fig. 7 (a). The hollow casing was also used to hide an MPPT board, lithium-ion battery, servo motor, microcontroller and wireless communication board. As with the TiltTile modules, a discreet touch sensitive switch is also mounted on the casing to provide user input.

\subsection{Capabilities and limitations}

Both the TiltTile and FabricOn devices harvest energy in low-power operation mode, keeping the microcontroller (normally $1.2 \mu \mathrm{A}$ ), servo motor (normally $0 \mu \mathrm{A}$ ) and wireless communication module (normally $1 \mu \mathrm{A}$ ) in sleep mode wherever possible. For receiving messages, the TiltTile is operated in cyclic sleep mode - waking up for 1 second every 15 minutes to check/receive messages. To send messages, it wakes up when the user touches the device's switch. The two demonstrator devices have different messaging capacities due to the different PV technologies employed.

8.3.1 TiltTile messaging capacity. We measured the energy harvesting performance of our DSSC cells under two different indoor illumination conditions: bright light near a south-facing window (max. $5000 \mathrm{~lx}$ ), and lower light away from a window (max. 1000 lx) over a 12-hour period of daytime. The average energy levels produced were $32 \mathrm{~mW} \mathrm{~h}$ and $16 \mathrm{~mW} \mathrm{~h}$, respectively. Received light intensity is reduced by different amounts on each side of the DSSC module when it is in the open state (i.e., facing sideways) - in this position the average energy harvested is about $15 \%$ lower. With typical energy harvester efficiency of $\mathrm{m} 80 \%$ and each state rotation consuming $\mathrm{m} 0.23 \mathrm{~mW} \mathrm{~h}$, we estimate that up to four TiltTile interactions per hour are possible; i.e., around 48 per 12-hour day.

8.3.2 FabricOn messaging capacity. We measured the total energy harvested by an OPV tape over a 12-hour period in the same ambient lighting conditions as in the previous section. In the 5000 lx condition our test module produced up to $9 \mathrm{~mW} \mathrm{~h}$. The OPV tape's performance is far poorer in low light intensity, producing only about $15 \%$ of that in the bright light condition. Each FabricOn interaction consumes $\mathrm{m} 1.42 \mathrm{~mW}$ h, so we estimate that it may be possible to interact with the roller approximately five times per 12-hour day when positioned in bright light.

8.3.3 Limitations. The main limitation of the current prototypes is, as might be expected, their energy use and generation capability. This is a natural limitation of self-powered devices, but there are several potential options for improvement. Using a different PV technology could improve efficiency, particularly in low light conditions. For example, OPV cells have limited conversion efficiency of only around $4 \%$ in direct sunlight. One alternative could be to use perovskite cells, which can achieve a higher efficiency of 27 $\%$ [22] while offering similar cost, flexible form factor and the ability to create aesthetic colours and patterns for further blending into nearby surfaces.

Both demonstrator devices use servo motors to rotate or roll between states. In the current versions we have integrated the smallest possible servo motors that could rotate or roll the PV modules. The energy consumption, size and weight of these motors currently limits the respective parameters of a PV-Pix device. Other mechanisms, such as electrostatic actuators, could be integrated to reduce energy consumption and weight. Similarly, our existing PV-Pix prototypes use direct wireless communication to send and receive messages. While this is feasible between closely-located families in Dharavi, this approach will consume higher energy to communicate over a longer range. Significant energy could be saved by, for example, communicating through a nearby smartphone where possible. While the use of a smartphone might appear to make the PV-Pix approach redundant-i.e., why not simply send messages via smartphones?-we note that the form factor and communication possibilities enabled by our approach are not well served by a mobile (e.g., the reluctance of elders in households to use smartphones). 

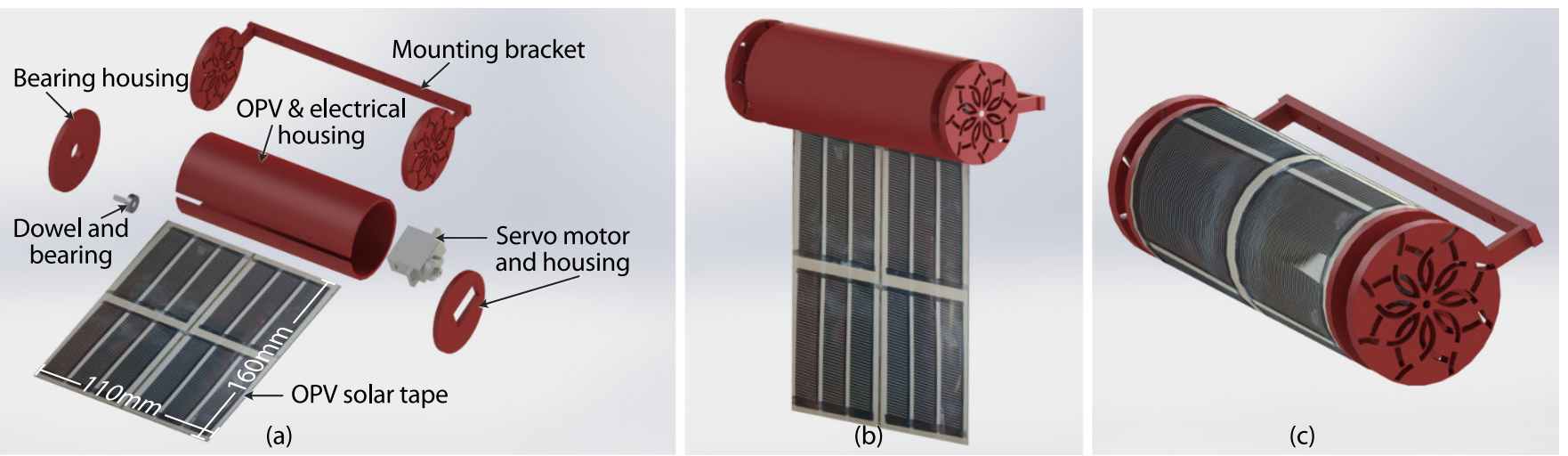

Figure 7: PV-Pix prototype 2 - FabricOn. This prototype is constructed using a commercially available flexible organic photovoltaic (OPV) solar-cell, a servo motor and a series of 3D-printed and laser-cut housings (a), and rolls up or down to change between the (b) open and (c) closed states. Note that as in Fig. 6 we use rendered diagrams of the prototype for clarity here, and the accompanying video figure shows working examples of the actual prototype in use.

\subsection{Reflections from community participants on the PV-Pix concept}

It was not our intention to carry out an evaluation of the working prototypes due to their experimental nature. However, it was important to provide the participants with an opportunity to at least see the technologies that they had helped create. Due to Covid-19 restrictions we could not take physical objects to participants, but created videos such as the one accompanying this paper for them to view on their mobile phones. They could relate their low-fidelity experiences with the high-fidelity embodiments, but wanted to be able to use a full working version. They saw the progress made on the working version as a commitment to the idea and expressed optimism that this could help people across India.

\section{CONCLUSIONS AND FUTURE WORK}

By working alongside co-designers from slum communities in Mumbai, over an extended period with a diverse set of activities, together we have elaborated a new class of material for inter-location communication and explored its uses in situ. The material, which we call PV-Pix, self-powers deformations by harvesting light energy to afford messaging between homes. While there have been other uses of PV to power digital interfaces and interactions, our work emphasises the value of re-seeing such propositions through the settings, experiences and the imaginations of communities that are usually marginalised from mainstream future-envisioning. While the resulting material and its use is demonstrated in slum homeshomes that are far from those often portrayed for instance in glossy technology advertising-we posit its relevance and potential to act as an extensible computational material in far more affluent and technology-rich environments (be they in California, Tokyo, London or the million dollar residences that overlook Dharavi). A key next step, then, is to deploy working prototypes of PV-Pixconstructed displays in both emergent and mainstream user homes over an extended period to both observe and compare evolving practices, as well as to provoke alternative PV-Pix designs that draw on the design space we have presented. We provide a toolkit to accompany this paper ${ }^{7}$ in order that other researchers can join us on this research journey.

\section{ACKNOWLEDGMENTS}

We would like to thank the following people for their contribution to this work: Manik and the workshop participants in Dharavi; Mrs Latha Vishwanath, Mr Mohan Kollur, Mr Shivshankar Doddamani, Mr Chandrakant and their respective families; Subhod Poddar, Saurabh Seth and Aparna Kulkarni for their artistic perspective; Jose Raju for assistance with the accompanying video as well as lowfidelity prototyping; and, Hanif Galwani and the other craftsmen associated with Galwani pottery studio, Kumbharwada, Dharavi. This work was supported by Engineering and Physical Sciences Research Council grants EP/R032750/1 (https://deliot.me) and EP/N013948/1 (https://breaking-the-glass.com), and Welsh European Funding Office SO1.1 Operation SPARC-II (https://swansea.ac.uk/sparc-ii/).

\section{REFERENCES}

[1] Gregory D. Abowd. 2020. The Internet of Materials: A Vision for Computational Materials. IEEE Pervasive Computing 19, 2 (2020), 56-62. https://doi.org/10.1109/ MPRV.2020.2982475

[2] Nivedita Arora, Steven L. Zhang, Fereshteh Shahmiri, Diego Osorio, Yi-Cheng Wang, Mohit Gupta, Zhengjun Wang, Thad Starner, Zhong Lin Wang, and Gregory D. Abowd. 2018. SATURN: A Thin and Flexible Self-powered Microphone Leveraging Triboelectric Nanogenerator. Proceedings of the ACM on Interactive, Mobile, Wearable and Ubiquitous Technologies 2, 2, Article 60 (2018), 28 pages. https://doi.org/10.1145/3214263

[3] Jeb Brugman. 2013. The Making of Dharavi's 'Citysystem'. In Dharavi: The City Within, Joseph Campana (Ed.). Harper Collins India, New Delhi.

[4] Joseph Campana (Ed.). 2013. Dharavi: The City Within. Harper Collins India, New Delhi.

[5] Arunkumar Chandrasekhar, Nagamalleswara Rao Alluri, Venkateswaran Vivekananthan, Jung Hwan Park, and Sang-Jae Kim. 2017. Sustainable Biomechanical Energy Scavenger toward Self-Reliant Kids' Interactive Battery-Free Smart Puzzle. ACS Sustainable Chemistry \& Engineering 5, 8 (2017), 7310-7316. https://doi.org/10.1021/acssuschemeng.7b01561

[6] Ayona Datta. 2016. Fast Cities in an Urban Age. In Mega-Urbanization in the Global South: Fast Cities and New Urban Utopias of the Postcolonial State, Ayona Datta and Abdul Shaban (Eds.). Routledge, London, UK.

[7] Devanuj and Anirudha Joshi. 2013. Technology Adoption by 'emergent' Users: The User-Usage Model. In Proceedings of the 11th Asia Pacific Conference on

\footnotetext{
${ }^{7}$ See: https://github.com/fitlab-swansea/pv-pix
} 
Computer Human Interaction (APCHI '13). Association for Computing Machinery, New York, NY, USA, 28-38. https://doi.org/10.1145/2525194.2525209

[8] Tobias Grosse-Puppendahl, Steve Hodges, Nicholas Chen, John Helmes, Stuart Taylor, James Scott, Josh Fromm, and David Sweeney. 2016. Exploring the Design Space for Energy-Harvesting Situated Displays. In Proceedings of the 29th Annual Symposium on User Interface Software and Technology (UIST '16). Association for Computing Machinery, New York, NY, USA, 41-48. https://doi.org/10.1145/ 2984511.2984513

[9] Lars Hallnäs and Johan Redström. 2001. Slow Technology - Designing for Reflection. Personal and Ubiquitous Computing 5, 3 (2001), 201--212. https //doi.org/10.1007/PL00000019

[10] Richard Harper. 2010. Texture: Human Expression in the Age of Communications Overload. MIT Press, Cambridge, MA.

[11] Marc Hassenzahl, Stephanie Heidecker, Kai Eckoldt, Sarah Diefenbach, and Uwe Hillmann. 2012. All You Need is Love: Current Strategies of Mediating Intimate Relationships through Technology. ACM Transactions on Computer-Human Interaction 19, 4, Article 30 (2012), 19 pages. https://doi.org/10.1145/2395131. 2395137

[12] Matt Jones, Simon Robinson, Jennifer Pearson, Manjiri Joshi, Dani Raju, Charity Chao Mbogo, Sharon Wangari, Anirudha Joshi, Edward Cutrell, and Richard Harper. 2017. Beyond "Yesterday's Tomorrow": Future-Focused Mobile Interaction Design by and for Emergent Users. Personal and Ubiquitous Computing 21, 1 (2017), 157-171. https://doi.org/10.1007/s00779-016-0982-0

[13] Vivek Kant and Anirudha Joshi. 2018. Challenges In Supporting The Emergent User. In Proceedings of the 9th Indian Conference on Human Computer Interaction (IndiaHCI '18). Association for Computing Machinery, New York, NY, USA, 67-70. https://doi.org/10.1145/3297121.3297131

[14] Aditya Kundalkar. 2013. Potters, Sailors and Financiers. In Dharavi: The City Within, Joseph Campana (Ed.). Harper Collins India, New Delhi.

[15] Dominik Landerer, Daniel Bahro, Holger Röhm, Manuel Koppitz, Adrian Mertens, Felix Manger, Fabian Denk, Michael Heidinger, Thomas Windmann, and Alexander Colsmann. 2017. Solar Glasses: A Case Study on Semitransparent Organic Solar Cells for Self-Powered, Smart, Wearable Devices. Energy Technology 5, 11 (2017), 1936-1945. https://doi.org/10.1002/ente.201700226

[16] John Law. 2004. After Method: Mess in Social Science Research. Routledge, Oxford, UK.

[17] Mathieu Le Goc, Lawrence H. Kim, Ali Parsaei, Jean-Daniel Fekete, Pierre Dragicevic, and Sean Follmer. 2016. Zooids: Building Blocks for Swarm User Interfaces In Proceedings of the 29th Annual Symposium on User Interface Software and Technology (UIST '16). Association for Computing Machinery, New York, NY, USA 97-109. https://doi.org/10.1145/2984511.2984547

[18] Yanki Lee. 2008. Design participation tactics: the challenges and new roles for designers in the co-design process. CoDesign 4, 1 (2008), 31-50. https: //doi.org/10.1080/15710880701875613

[19] Andrés Lucero, Kirsikka Vaajakallio, and Peter Dalsgaard. 2012. The dialogue-labs method: process, space and materials as structuring elements to spark dialogue in co-design events. CoDesign 8, 1 (2012), 1-23. https://doi.org/10.1080/15710882. 2011.609888

[20] David Martineau. 2012. Dye Solar Cells for Real: The Assembly Guide for Making Your Own Solar Cells. Solaronix. https://www.solaronix.com/documents/dye solar_cells_for_real.pdf

[21] Yogesh Kumar Meena, Krishna Seunarine, Deepak Ranjan Sahoo, Simon Robinson, Jennifer Pearson, Chi Zhang, Matt Carnie, Adam Pockett, Andrew Prescott, Suzanne K. Thomas, Harrison Ka Hin Lee, and Matt Jones. 2020. PV-Tiles Towards Closely-Coupled Photovoltaic and Digital Materials for Useful, Beautiful and Sustainable Interactive Surfaces. In Proceedings of the 2020 CHI Conference on Human Factors in Computing Systems (CHI '20). Association for Computing Machinery, New York, NY, USA, Article 241, 12 pages. https://doi.org/10.1145/ 3313831.3376368

[22] Pabitra K Nayak, Suhas Mahesh, Henry J Snaith, and David Cahen. 2019. Photovoltaic solar cell technologies: analysing the state of the art. Nature Reviews Materials 4, 4 (2019), 269-285. https://doi.org/10.1038/s41578-019-0097-0

[23] Deepak Ranjan Padhi, Anirudha Joshi, Abhishek Shrivastava, and Rucha Tulaskar. 2018. Hierarchy or List? Comparing Menu Navigation by Emergent Users. In Proceedings of the 9th Indian Conference on Human Computer Interaction (IndiaHCI '18). Association for Computing Machinery, New York, NY, USA, 29-34. https: //doi.org/10.1145/3297121.3297125

[24] Jennifer Pearson, Simon Robinson, and Matt Jones. 2016. Exploring Low-Cost, Internet-Free Information Access for Resource-Constrained Communities. ACM Transactions on Computer-Human Interaction 23, 6, Article 36 (2016), 34 pages. https://doi.org/10.1145/2990498

[25] Jennifer Pearson, Simon Robinson, Thomas Reitmaier, Matt Jones, and Anirudha Joshi. 2019. Diversifying Future-Making Through Itinerative Design. ACM Transactions on Computer-Human Interaction 26, 5, Article 33 (2019), 21 pages. https://doi.org/10.1145/3341727

[26] Dani Kalarikalayil Raju. 2018. Participatory Design for Creating Virtual Environments. In Proceedings of the 9th Indian Conference on Human Computer Interaction (IndiaHCI '18). Association for Computing Machinery, New York, NY, USA, 63-66. https://doi.org/10.1145/3297121.3297129

[27] Simon Robinson, Jennifer Pearson, Thomas Reitmaier, Shashank Ahire, and Matt Jones. 2018. Make Yourself at Phone: Reimagining Mobile Interaction Architectures With Emergent Users. In Proceedings of the 2018 CHI Conference on Human Factors in Computing Systems (CHI '18). ACM, New York, NY, USA, Article 407, 12 pages. https://doi.org/10.1145/3173574.3173981

[28] Nithya Sambasivan, Ed Cutrell, Kentaro Toyama, and Bonnie Nardi. 2010. Intermediated Technology Use in Developing Communities. In Proceedings of the SIGCHI Conference on Human Factors in Computing Systems (CHI '10). Association for Computing Machinery, New York, NY, USA, 2583-2592. https: //doi.org/10.1145/1753326.1753718

[29] Douglas Schuler and Aki Namioka. 1993. Participatory design: Principles and practices. CRC Press, Boca Raton, FL, USA.

[30] Donald A. Schön. 1992. Designing as Reflective Conversation with the Materials of a Design Situation. Research in Engineering Design 3, 3 (1992), 131-147. https: //doi.org/10.1007/BF01580516

[31] Susanne Seitinger, Daniel S. Perry, and William J. Mitchell. 2009. Urban Pixels: Painting the City with Light. In Proceedings of the SIGCHI Conference on Human Factors in Computing Systems (CHI '09). Association for Computing Machinery, New York, NY, USA, 839-848. https://doi.org/10.1145/1518701.1518829

[32] Fereshteh Shahmiri, Chaoyu Chen, Anandghan Waghmare, Dingtian Zhang, Shivan Mittal, Steven L. Zhang, Yi-Cheng Wang, Zhong Lin Wang, Thad E. Starner, and Gregory D. Abowd. 2019. Serpentine: A Self-Powered Reversibly Deformable Cord Sensor for Human Input. In Proceedings of the 2019 CHI Conference on Human Factors in Computing Systems (CHI '19). Association for Computing Machinery, New York, NY, USA, Article 545, 14 pages. https://doi.org/10.1145/3290605. 3300775

[33] Roger Silverstone and Leslie Haddon. 1996. Design and the domestication of information and communication technologies: Technical change and everyday life. In Communication by Design: The Politics of Information and Communication Technologies, Robin Mansell and Roger Silverstone (Eds.). Oxford University Press, Oxford, UK, 44-74.

[34] Varun Sivaram. 2018. Taming the Sun: Innovations to Harness Solar Energy and Power the Planet. The MIT Press, Cambridge, MA, USA.

[35] Lucy Suchman. 2002. Practice-Based Design of Information Systems: Notes from the Hyperdeveloped World. The Information Society 18, 2 (2002), 139-144. https://doi.org/10.1080/01972240290075066

[36] Anran Wang, Vikram Iyer, Vamsi Talla, Joshua R Smith, and Shyamnath Gollakota. 2017. FM Backscatter: Enabling Connected Cities and Smart Fabrics. In 14th USENIX Symposium on Networked Systems Design and Implementation (NSDI '17). USENIX Association, Boston, MA, USA, 243-258.

[37] Laura Watts. 2018. Energy at the End of the World: An Orkney Islands Saga. The MIT Press, Cambridge, MA, USA.

[38] Laura Watts, James Auger, and Julian Hanna. 2018. The Newton Machine: Reconstrained Design for Energy Infrastructure. In Control, Change and CapacityBuilding in Energy Systems., Patrick Sumpf and Christian Büscher (Eds.). Shape Energy, Cambridge, UK.

[39] Zhen Wen, Min-Hsin Yeh, Hengyu Guo, Jie Wang, Yunlong Zi, Weidong Xu, Jianan Deng, Lei Zhu, Xin Wang, Chenguo Hu, et al. 2016. Self-powered textile for wearable electronics by hybridizing fiber-shaped nanogenerators, solar cells, and supercapacitors. Science Advances 2, 10, Article e1600097 (2016), 8 pages. https://doi.org/10.1126/sciadv.1600097

[40] Dingtian Zhang, Jung Wook Park, Yang Zhang, Yuhui Zhao, Yiyang Wang, Yunzhi Li, Tanvi Bhagwat, Wen-Fang Chou, Xiaojia Jia, Bernard Kippelen, Canek FuentesHernandez, Thad Starner, and Gregory D. Abowd. 2020. OptoSense: Towards Ubiquitous Self-Powered Ambient Light Sensing Surfaces. Proceedings of the ACM on Interactive, Mobile, Wearable and Ubiquitous Technologies 4, 3, Article 103 (2020), 27 pages. https://doi.org/10.1145/3411826

[41] John Zimmerman, Erik Stolterman, and Jodi Forlizzi. 2010. An Analysis and Critique of Research through Design: Towards a Formalization of a Research Approach. In Proceedings of the 8th ACM Conference on Designing Interactive Systems (DIS '10). Association for Computing Machinery, New York, NY, USA, 310-319. https://doi.org/10.1145/1858171.1858228 